\title{
Hybrid Fixed Point Theorem with Applications to Forced Damped Oscillations and Infinite Systems of Fractional Order Differential Equations
}

\author{
Muhammad Shoaib $\mathbb{D}^{1}$, Muhammad Sarwar $\mathbb{D}^{1},{ }^{1}$ and Thabet Abdeljawad $\mathbb{D}^{2,3,4}$ \\ ${ }^{1}$ Department of Mathematics, University of Malakand, Chakdara Dir (L), Pakistan \\ ${ }^{2}$ Department of Mathematics and General Sciences, Prince Sultan University, P.O. Box 66833, Riyadh 11586, Saudi Arabia \\ ${ }^{3}$ Department of Medical Research, China Medical University, Taichung 40402, Taiwan \\ ${ }^{4}$ Department of Computer Science and Information Engineering, Asia University, Taichung, Taiwan
}

Correspondence should be addressed to Muhammad Sarwar; sarwarswati@gmail.com

and Thabet Abdeljawad; tabdeljawad@psu.edu.sa

Received 9 April 2020; Revised 11 August 2020; Accepted 24 September 2020; Published 8 December 2020

Academic Editor: Antonio Francisco Roldan Lopez de Hierro

Copyright (C) 2020 Muhammad Shoaib et al. This is an open access article distributed under the Creative Commons Attribution License, which permits unrestricted use, distribution, and reproduction in any medium, provided the original work is properly cited.

\begin{abstract}
In this manuscript, hybrid common fixed point results in the setting of a $b$-metric space are established. Our results generalized the results of Fisher, Khan, and Piri et al. for set-valued mapping in $b$-metric spaces. Applications to forced damped oscillations, infinite systems of fractional order differential equations, and system of functional equations are also studied. We construct an example to support our main result.
\end{abstract}

\section{Introduction and Preliminaries}

The idea of a metric space was generalized by Czerwik [1] and Bakhtin [2]. They presented metric spaces called $b$-metric spaces. Several researchers took the idea of Czerwik and illustrated interesting results. For details, see [3-7]. For recent generalizations to $b$-metric spaces by employing control functions in the triangle inequality to replace the constant of the $b$-metric triangle inequality, we refer to [8-12] and the references therein.

In 1973, the contraction was introduced by Geraghty [13] in which the contraction constant was quite changed by mapping owing to its interesting properties. After that, several papers for rational Geraghty contractive mappings have appeared (for details, see [14-18]). Khan [19] introduced one of the best works in this line, and Fisher [20] modified it. By rational expressions, Khan [19] and Fisher [20] results were lately extended by Piri et al. [21] by introducing a new general contractive condition. Fixed point results via $F$-Khan contractions were studied by Piri et al. [22] on complete metric spaces, and they discuss their application to integral equations. In [23], Ullah et al. established fixed point results and discuss the application to an infinite system of fractional order differential equations.

Nadler [24] elaborated and extended the Banach contraction principle [25] to set-valued mapping by using the Hausdorff metric. After different generalizations of the Nadler contraction principle, Wardowski [26] introduced a contraction called $F$-contraction. In this way, Wardowski generalized the Banach contraction principle (BCP) in a different manner from the known results of literature. Following this direction, Sgroi and Vetro [27] studied set-valued F-contractions and discussed their application on certain functional and integral equations.

Cosentino and Vetro [25] extended F-contraction in the setting of $b$-metric spaces and proved some fixed point results. Ali et al. [28] studied the fixed point, generalize the result of Cosentino et al. [25] for a new class of $F$-contractions in the set- 
ting of $b$-metric spaces, and apply the result to obtain existence results for Volterra-type integral inclusion in $b$-metric spaces. Several authors generalized $F$-contraction by combining it with some existing contractive conditions (see [27, 29-32]).

In the current work, we derive a hybrid (single and multivalued) common fixed point result for the F-Khan-type contraction in the $b$-metric space. Also, we shall provide an example and applications for the validity of the established result. Throughout this paper, $C B(\Lambda)$ indicates the family of nonempty subsets of $\Lambda$, which is bounded and closed. $\mathbb{R}^{+}, \mathbb{N}_{0}$, and $\mathbb{N}$ signify the set of any nonnegative real numbers, the set of nonnegative integers, and the set of positive integers. Now, we recall a few basic results and definitions.

Definition 1 [1]. Consider a nonempty set $\Lambda$ and let $s \geq 1$. Assume $d: \Lambda \times \Lambda \longrightarrow \mathbb{R}^{+}$is a function satisfying the conditions:

(1) $d\left(\xi_{11}, \xi_{22}\right)=0 \Leftrightarrow \xi_{11}=\xi_{22}$ for all $\xi_{11}, \xi_{22} \in \Lambda$

(2) $d\left(\xi_{11}, \xi_{22}\right)>0, \xi_{11} \neq \xi_{22}$, for all $\xi_{11}, \xi_{22} \in \Lambda$

(3) $d\left(\xi_{11}, \xi_{22}\right)=d\left(\xi_{22}, \xi_{11}\right)$, where $\xi_{11}, \xi_{22} \in \Lambda$

(4) $d\left(\xi_{11}, \xi_{22}\right) \leq s\left(d\left(\xi_{11}, \xi_{33}\right)+d\left(\xi_{33}, \xi_{22}\right)\right)$ for all $\xi_{11}$, $\xi_{22}, \xi_{33} \in \Lambda$

Then, the triple $(\Lambda, d, s)$ is called the $b$-metric space.

Definition 2 [1]. Assume $(\Lambda, d, s)$ is a $b$-metric space, where $s \geq 1$. Let $\sigma_{n}$ be a sequence in $\Lambda$. Then, $\sigma \in \Lambda$ is said to be the limit of the sequence $\sigma_{n}$ if

$$
\lim _{n \rightarrow \infty} d(\sigma n, \sigma)=0
$$

and the sequence $\sigma_{n}$ is said to be convergent in $\Lambda$.

Definition 3 [1]. If for each $\epsilon>0$, there is a positive integer $\mathbb{N}$ such that $d\left(\sigma_{n}, \sigma_{m}\right)<\epsilon$ for all $n, m>\mathbb{N}$, then a sequence $\sigma_{n}$ is said to be a $b$-Cauchy sequence.

Definition 4 [1]. A $b$-metric space $(\Lambda, d, s)$ is said to be complete (or a $b$-complete metric space) if every Cauchy sequence in $(\Lambda, d, s)$ is convergent in $\Lambda$.

Definition 5 [33]. Assume $s \geq 1$ is a real number and $\mathbb{F}$ represents the family of functions $F: \mathbb{R}^{+} \longrightarrow \mathbb{R}$, with the below conditions:

$\left(F_{1}\right)$ For each $\left\{\gamma_{n}\right\} \subset \mathbb{R}^{+}$which is a positive term sequence, $F$ is strictly increasing.

$\left(F_{2}\right) \lim _{n \rightarrow \infty} \gamma_{n}=0 \Leftrightarrow \lim _{n \rightarrow \infty} F\left(\gamma_{n}\right)=\infty$.

$\left(F_{3}\right)$ For each positive number sequence, i.e., $\left\{\gamma_{n}\right\} \subset$ $\mathbb{R}^{+}, \lim _{n \rightarrow \infty} \gamma_{n}=0$, there exists $k \in(0,1)$ such that $\lim _{n \rightarrow \infty}\left(\gamma_{n}\right)^{k}$ $F\left(\gamma_{n}\right)=0$

$\left(F_{4}\right)$ For $\left\{\gamma_{n}\right\} \subset \mathbb{R}^{+}$, such that $\lambda+F\left(s \gamma_{n}\right) \leq F\left(\gamma_{n-1}\right) \forall n \in$ $\mathbb{N}$ and some $\lambda \in \mathbb{R}^{+}$, then $\lambda+F\left(s^{n} \gamma_{n}\right) \leq F\left(s^{n-1} \gamma_{n-1}\right)$.

Now, we give some basics defined in a $b$-metric space $(\Lambda, d, s)$ for set-valued mappings. Define the mapping $\mathbb{H}: C$ $B(\Lambda) \times C B(\Lambda) \longrightarrow \mathbb{R}^{+}$for $\Lambda_{1}, \Lambda_{2} \in C B(\Lambda)$ by

$$
\mathbb{H}\left(\Lambda_{1}, \Lambda_{2}\right)=\max \left\{\delta\left(\Lambda_{1}, \Lambda_{2}\right), \delta\left(\Lambda_{2}, \Lambda_{1}\right)\right\}
$$

where

$$
\begin{gathered}
\delta\left(\Lambda_{1}, \Lambda_{2}\right)=\sup \left\{d\left(a, \Lambda_{2}\right), a \in \Lambda_{1}\right\} \\
\delta\left(\Lambda_{2}, \Lambda_{1}\right)=\sup \left\{d\left(b, \Lambda_{1}\right), b \in \Lambda_{1}\right\}, \\
d(a, \Lambda)=\inf \left\{d\left(a, \sigma_{1}\right), \sigma_{1} \in \Lambda\right\}
\end{gathered}
$$

Lemma $6[16,34]$. Assume a $b$-metric space $(\Lambda, d, s)$. Suppose $\Lambda_{1}, \Lambda_{2}, \Lambda_{3} \in C B(\Lambda)$ and $\sigma_{1}, \sigma_{2} \in \Lambda$; then, the following holds:

(1) $d\left(\sigma_{1}, \Lambda_{2}\right) \leq d\left(\sigma_{1}, b\right)$ for any $b \in \Lambda_{2}$

(2) $\delta\left(\Lambda_{1}, \Lambda_{2}\right) \leq \mathbb{H}\left(\Lambda_{1}, \Lambda_{2}\right)$

(3) $d(\sigma 1, \Lambda 2) \leq \mathbb{H}\left(\Lambda_{1}, \Lambda_{2}\right)$ for any $\sigma 1 \in \Lambda_{1}$

$$
\mathbb{H}\left(\Lambda_{1}, \Lambda_{1}\right)=0,
$$

(4) $\mathbb{H}\left(\Lambda_{1}, \Lambda_{2}\right)=\mathbb{H}\left(\Lambda_{2}, \Lambda_{1}\right)$

$$
\begin{aligned}
& \mathbb{H}\left(\Lambda_{1}, \Lambda_{3}\right) \leq s\left[\mathbb{H}\left(\Lambda_{1}, \Lambda_{2}\right)+\mathbb{H}\left(\Lambda_{2}, \Lambda 3\right)\right] \\
& \mathbb{H}\left(\sigma_{1}, \Lambda_{1}\right) \leq s\left[d\left(\sigma_{1}, \sigma_{2}\right)+d\left(\sigma_{2}, \Lambda_{1}\right)\right] .
\end{aligned}
$$

Lemma $7[20,24]$. Let $(\Lambda, d)$ be a metric space. Further, $\Lambda_{1}$, $\Lambda_{2} \in C B(\Lambda)$; then, for $\delta \geq 1$ and for each $\zeta_{1} \in \Lambda_{1}$, there exists $\zeta_{2}\left(\zeta_{1}\right) \in \Lambda_{2}$ such that $d\left(\zeta_{1}, \zeta_{2}\right) \leq \delta \mathbb{H}\left(\Lambda_{1}, \Lambda_{2}\right)$.

Lemma $8[16,34]$. Assume a b-metric space $(\Lambda, d, s)$ and $\Lambda_{1}, \Lambda_{2} \in C B(\Lambda)$. Then, for $\delta>1, \zeta_{1} \in \Lambda_{1}$, there exists $\zeta_{2}\left(\zeta_{1}\right)$ $\in \Lambda_{2}$ such that $d\left(\zeta_{1}, \zeta_{2}\right) \leq \delta \mathrm{H}\left(\Lambda_{1}, \Lambda_{2}\right)$.

Theorem 9 [24]. Assume $\lambda: \Lambda \longrightarrow C B(\Lambda)$ is a multivalued mapping on a complete metric space $(\Lambda, d)$ such that

$$
\mathbb{H}\left(\lambda \sigma_{1}, \lambda \sigma_{2}\right) \leq \mu\left(d\left(\sigma_{1}, \sigma_{2}\right)\right), \quad \forall \sigma_{1}, \sigma_{2} \in \Lambda,
$$

where $\mu \in(0,1)$. Then, $\lambda$ has a fixed point.

Theorem 10 [25]. Let $(\Lambda, d, s)$ be a b-metric space, where $s$ $\geq 1$. For $F \in \mathbb{F}$, a multivalued mapping $\lambda: \Lambda \longrightarrow C B(\Lambda)$ is called a Nadler-type F-contraction such that for $\tau \in \mathbb{R}^{+}$,

$$
2 \tau+F\left(s \mathbb{H}\left(\lambda \xi_{1}, \lambda \xi_{2}\right)\right) \leq F\left(d\left(\xi_{1}, \xi_{2}\right)\right) .
$$

$\forall \xi_{1}, \xi_{2} \in \Lambda \lambda \xi_{1} \neq \lambda \xi_{2}$; then, $\lambda$ has a fixed point in $\Lambda$.

\section{Main Results}

Definition 11. Let $(\Lambda, d, s)$ be a $b$-metric space. Let $\theta: \Lambda$ $\longrightarrow \Lambda$ and $\Theta: \Lambda \longrightarrow C B(\Lambda)$ be two mappings. Then, $\Theta$ is said to be $F$-Khan contraction w.r.t $\theta$ if there exists $0<\tau<$ $\infty$ and $F \in \mathbb{F}$ such that for all $\xi, \zeta \in \Lambda, \max \{d(\xi, \theta \zeta), d(\theta \xi$, $\zeta)\} \neq 0$; then, $\theta \xi \neq \theta \zeta$ and 


$$
\tau+F(s \mathbb{H}(\theta \xi, \theta \zeta)) \leq \begin{cases}F\left(\frac{d(\xi, \theta \xi) d(\xi, \theta \zeta)+d(\zeta, \theta \zeta) d(\zeta, \theta \xi)}{\xi\{d(\xi, \theta \zeta), d(\theta \xi, \zeta)\}}\right), & \text { if } \max \{d(\xi, \theta \zeta), d(\theta \xi, \zeta)\} \neq 0 \\ 0, & \text { if } \max \{d(\xi, \theta \zeta), d(\theta \xi, \zeta)\}=0\end{cases}
$$

Theorem 12. Assume $(\Lambda, d, s)$ is a complete b-metric space. Let $(\theta, \Theta)$ be the F-Khan contraction. Further, $\theta$ is continuous. Then, $C(\theta, \Theta) \neq \varnothing$.

(i) $\theta$ and $\Theta$ have a common fixed point if $\theta \theta \alpha=\theta \alpha$, and $\theta$ is occasionally $\Theta$-weakly commuting at $\alpha$. Then, $\theta$ and $\Theta$ have a common fixed point
Proof. Let $\zeta_{0} \in \Lambda$ and $\zeta_{1} \in \Theta \zeta_{0}$. Let $\zeta_{2}:=\theta \zeta_{1}$. By Lemma 8, there exists $\zeta_{3} \in \Theta \zeta_{2}$ such that $d\left(\zeta_{3}, \zeta_{2}\right) \leq \mathbb{H}\left(\Theta \zeta_{2},\left\{\theta \zeta_{1}\right\}\right)$. Inductively, we let $\zeta_{2 n}:=\theta \zeta_{2 n-1}$, and by Lemma 8 , we choose $\zeta_{2 n+1} \in \Theta \zeta_{2 n}$ such that

$$
d\left(\zeta_{2 n-1}, \zeta_{2 n}\right) \leq \mathbb{H}\left(\Theta \zeta_{2 n},\left\{\theta \zeta_{2 n-1}\right\}\right) .
$$

Using equation (8), we have

$$
\begin{aligned}
\tau+ & F\left(s \mathbb{H}\left(\Theta \zeta_{2 n}, \Theta \zeta_{2 n+1}\right)\right) \\
& \leq F\left(\left\{\frac{d\left(\zeta_{2 n}, \theta \zeta_{2 n}\right) d\left(\zeta_{2 n}, \theta \zeta_{2 n+1}\right)+d\left(\zeta_{2 n+1}, \theta \zeta_{2 n+1}\right) d\left(\zeta_{2 n+1}, \theta \zeta_{2 n}\right)}{\max \left\{d\left(\theta \zeta_{2 n}, \zeta_{2 n+1}\right), d\left(\zeta_{2 n}, \theta \zeta_{2 n+1}\right)\right\}}\right\}\right) \\
& \leq F\left(d\left(\zeta_{2 n}, \theta \zeta_{2 n}\right)\right),
\end{aligned}
$$

which implies

$$
\tau+F\left(s \mathbb{H}\left(\Theta \zeta_{2 n}, \Theta \zeta_{2 n+1}\right)\right) \leq F\left(d\left(\zeta_{2 n}, \theta \zeta_{2 n}\right)\right) .
$$

We deduce that

$$
\tau+F\left(s d\left(\zeta_{2 n+1}, \zeta_{2 n+2}\right)\right) \leq F\left(d\left(\zeta_{2 n}, \zeta_{2 n+1}\right)\right) .
$$

Let $Q_{n}=d\left(\zeta_{2 n+1}, \zeta_{2 n+2}\right)>0, \forall n \in \mathbb{N}$. It follows from (12) and axiom $F_{4}$ that

$$
\tau+F\left(s^{n} d\left(\zeta_{2 n+1}, \zeta_{2 n+2}\right)\right) \leq F\left(s^{n-1} d\left(\zeta_{2 n}, \zeta_{2 n+1}\right), \quad \forall n \in \mathbb{N}\right.
$$

Thus, by equation (13),

$$
\begin{gathered}
F\left(s^{n} Q_{n}\right) \leq F\left(s^{n-1} Q_{n-1}\right)-\tau, \\
F\left(s^{n-1} Q_{n-1}\right) \leq F\left(s^{n-2} Q_{n-2}\right)-2 \tau, \\
\vdots \\
F\left(s^{n} Q_{n}\right) \leq F\left(s^{o} Q_{0}\right)-n \tau,
\end{gathered}
$$

which implies that

$$
\lim _{n \rightarrow \infty} F\left(s^{n} Q_{n}\right)=-\infty
$$

By using $F_{2}$, we have

$$
\lim _{n \rightarrow \infty} s^{n} Q_{n}=0 .
$$

By the $F_{3}$ property, there exists $0<k<1$ such that

$$
\lim _{n \rightarrow \infty}\left(s^{n} Q_{n}\right)^{k} F\left(s^{n} Q_{n}\right)=0 .
$$

Equation (14) implies that

$$
F\left(s^{n} p_{n}\right) \leq F\left(p_{0}\right)-n \tau .
$$

Multiplying (18) by $\left(s^{n} p_{n}\right)^{k}$, we have

$$
\left(s^{n} p_{n}\right)^{k} F\left(s^{n} p_{n}\right) \leq\left(s^{n} p_{n}\right)^{k} F\left(p_{0}\right)-n \tau\left(s^{n} p_{n}\right)^{k},
$$

which implies that

$$
\left(s^{n} p_{n}\right)^{k} F\left(s^{n} p_{n}\right)-\left(s^{n} p_{n}\right)^{k} F\left(p_{0}\right) \leq-n \tau\left(s^{n} p_{n}\right)^{k} \leq 0 .
$$

Applying limit $n \longrightarrow 1$, we have

$$
\lim _{n \rightarrow \infty} n\left(s^{n} p_{n}\right)^{k}=0 .
$$

From (21), there exists $n_{1} \in \mathbb{N}$ such that $n\left(s^{n} Q_{n}\right)^{k}<1$ such that

$$
s^{n} Q_{n} \leq \frac{1}{n n^{1 / k}}, \quad \forall n \geq n_{1} .
$$

To show that $\left\{\zeta_{n}\right\}$ is a $b$-Cauchy sequence, consider $m$, $n \in \mathbb{N}$ such that $m>n>n_{1}$, using triangular inequality, and using (18), we have 


$$
\begin{aligned}
d\left(\zeta_{2 n}, \zeta_{2 m}\right) \leq & s d\left(\zeta_{2 n}, \zeta_{2 n+1}\right)+s^{2} d\left(\zeta_{2 n+1}, \zeta_{2 n+2}\right)+\cdots \\
& +s^{m-n} d\left(\zeta_{2 m-1}, \zeta_{2 m}\right),=s Q_{n-1}+s^{2} Q_{n}+\cdots \\
& +s^{m-n} Q_{m-2},=\sum_{j=n-1}^{m-2} s^{j-n+2} Q_{j} \\
\leq & \sum_{j=n-1}^{\infty} s^{j-n+2} Q_{j} \leq \sum_{j=n-1}^{\infty} s^{2-n} \frac{1}{j^{1 / k}}
\end{aligned}
$$

By taking limit, we get $d\left(\zeta_{n}, \zeta_{m}\right) \longrightarrow 0$. Hence, $\left\{\zeta_{n}\right\}$ is a $b$ -Cauchy sequence, but a $b$-metric space $(\Lambda, d, s)$ is a complete space so there exists $\zeta \in \Lambda$ such that $\zeta_{n} \longrightarrow \zeta$ as $n \longrightarrow \infty$.

The next step is to show that $\zeta$ is a common fixed point of the mapping $\Theta$ and $\theta$. We have

$$
d\left(\zeta_{2 n+2}, \Theta \zeta\right) \leq \mathbb{H}\left(\Theta \zeta_{2 n+1}, \Theta \zeta\right) \leq s \mathbb{H}\left(\Theta \zeta_{2 n+1}, \Theta \zeta\right)
$$

which implies that

$$
d\left(\zeta_{2 n+2}, \Theta \zeta\right) \leq s \mathbb{H}\left(\Theta \zeta_{2 n+1}, \Theta \zeta\right)
$$

Since $F$ is strictly increasing, therefore

$$
F\left(d\left(\zeta_{2 n+2}, \Theta \zeta\right)\right) \leq F\left(s \mathbb{H}\left(\Theta \zeta_{2 n+1}, \Theta \zeta\right)\right)
$$

Adding $\tau$ to both sides and using equation (7), we have

$$
\begin{aligned}
\tau+ & F\left(d\left(\zeta_{2 n+2}, \Theta \zeta\right)\right) \\
& \leq \tau+F\left(s \mathbb{H}\left(\Theta \zeta_{2 n+1}, \Theta \zeta\right)\right) \\
& \leq F\left(\left\{\frac{d\left(\zeta_{2 n}, \theta \zeta_{2 n}\right) d\left(\zeta_{2 n}, \theta \zeta\right)+d(\zeta, \theta \zeta) d\left(\zeta, \theta \zeta_{2 n}\right)}{\max \left\{d\left(\zeta_{2 n+1}, \theta \zeta\right), d\left(\theta \zeta_{2 n+1}, \zeta\right)\right\}}\right\}\right)
\end{aligned}
$$

Since $\tau \in \mathbb{R}^{+}$, we have

$$
\begin{aligned}
& F\left(d\left(\zeta_{2 n+2}, \Theta \zeta\right)\right) \\
& \quad \leq F\left(\left\{\frac{d\left(\zeta_{2 n}, \theta \zeta_{2 n}\right) d\left(\zeta_{2 n}, \theta \zeta\right)+d(\zeta, \theta \zeta) d\left(\zeta, \theta \zeta_{2 n}\right)}{\max \left\{d\left(\zeta_{2 n+1}, \theta \zeta\right), d\left(\theta \zeta_{2 n+1}, \zeta\right)\right\}}\right\}\right) .
\end{aligned}
$$

Since $F$ is strictly increasing, therefore

$d\left(\zeta_{2 n+2}, \Theta \zeta\right) \leq\left\{\frac{d\left(\zeta_{2 n}, \theta \zeta_{2 n}\right) d\left(\zeta_{2 n}, \theta \zeta\right)+d(\zeta, \theta \zeta) d\left(\zeta, \theta \zeta_{2 n}\right)}{\max \left\{d\left(\zeta_{2 n+1}, \theta \zeta\right), d\left(\theta \zeta_{2 n+1}, \zeta\right)\right\}}\right\}$

Applying limit $n \longrightarrow \infty$, we get

$$
d(\zeta, \Theta \zeta) \leq 0
$$

which implies $\zeta \in \Theta \zeta$.

But

$$
d\left(\zeta_{2 n+2}, \Theta \zeta\right)=d\left(\theta \zeta_{2 n+1}, \Theta \zeta\right)
$$

By taking limit and using continuity of $\theta$, we have

$$
d(\zeta, \Theta \zeta)=\lim _{n \rightarrow \infty} d\left(\theta \zeta_{2 n+1}, \Theta \zeta\right)=d(\theta \zeta, \Theta \zeta)
$$

which implies $\theta \zeta \in \Theta \zeta$. Since $\theta \theta \zeta=\theta \zeta$ and $\theta \zeta \in \Theta \theta \zeta$, therefore $\gamma=\theta \gamma \in \Theta \gamma$.

By putting $\theta=\Theta$ in Theorem 12, we get the following.

Corollary 13. Assume $(\Lambda, d, s)$ is a complete b-metric space. Let $\Theta$ be an F-Khan contraction. Then, $\Theta$ has a fixed point.

Consider the following class of function.

Definition 14. Assume $s \geq 1$ is a real number and $\mathbb{F}_{\uparrow}$ represents the family of functions $F: \mathbb{R}^{+} \longrightarrow \mathbb{R}$, which is strictly increasing.

Definition 15. Let $(\Lambda, d, s)$ be a $b$-metric space. Let $\theta: \Lambda$ $\longrightarrow \Lambda$ and $\Theta: \Lambda \longrightarrow C B(\Lambda)$ be two mapping. Then, $\Theta$ is said to be the $F$-Khan contraction w.r.t $\theta$ if there exists $0<$ $\tau<\infty$ and $F \in \mathbb{F}_{\uparrow}$ such that for all $\xi, \zeta \in \Lambda, \max \{d(\xi, \theta \zeta), d$ $(\theta \xi, \zeta)\} \neq 0$; then, $\theta \xi \neq \theta \zeta$ and

$$
\tau+F(s \mathbb{H}(\Theta \xi, \Theta \zeta)) \leq \begin{cases}F\left(\frac{d(\xi, \theta \xi) d(\xi, \theta \zeta)+d(\zeta, \theta \zeta) d(\zeta, \theta \xi)}{\xi\{d(\xi, \theta \zeta), d(\theta \xi, \zeta)\}}\right), & \text { if } \max \{d(\theta \xi, \zeta), d(\theta \xi, \zeta)\} \neq 0 \\ 0, & \text { if } \max \{d(\xi, \theta \zeta), d(\theta \xi, \zeta)\}=0\end{cases}
$$

Theorem 16. Assume $(\Lambda, d, s)$ is a complete b-metric space. Let $(\theta, \Theta)$ be an F-Khan contraction. Further, if $\theta$ is continuous, then $C(\theta, \Theta) \neq \varnothing$.

(i) $\theta$ and $\Theta$ have a common fixed point if $\theta \theta \alpha=\theta \alpha$, and $\theta$ is occasionally $\Theta$-weakly commuting at $\alpha$. Then, $\theta$ and $\Theta$ have a common fixed point
Remarks. Our result extended the results of

(i) Fisher [20] for set-valued mapping in the setting of b-metric spaces

(ii) Khan [19] for set-valued mapping in b-metric spaces

(iii) Piri et al. [21, 22] for set-valued mapping in b-metric spaces 
Example 17. Consider the sequence $\left\{\left\{S_{q}\right\}: q \in\{1,2 \cdots 100\}\right\}$ as follows:

$$
S_{1}=1.2, S 2=2.3, \cdots, S_{q}=q(q+1) .
$$

Let $\Lambda=\left\{S_{q}: q \in\{1,2, \cdots, 100\}\right\}$ and $d: \Lambda \times \Lambda \longrightarrow[0$, $\infty)$ be defined by

$$
d(\xi, \zeta)=\max \{\xi, \zeta\}^{2}, \quad \text { if } \xi \neq \zeta d(\xi, \zeta)=0 \text {, if } \xi=\zeta .
$$

Then, $(\Lambda, d, s)$ is a complete $b$-metric space.

Define the mapping $\Theta: \Lambda \longrightarrow C B(\Lambda)$ by

$$
\Theta\left(S_{1}\right)=\left\{S_{1}\right\}, \Theta\left(S_{q}\right)=\left\{S_{q-1}\right\},
$$

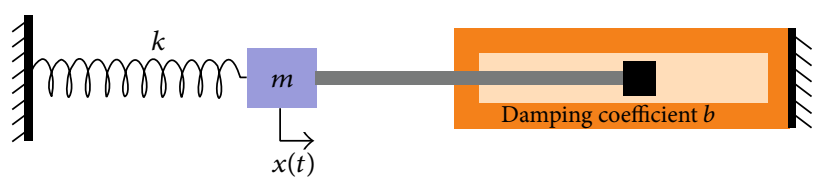

FIGURE 1: Forced oscillations.

and $\theta: \Lambda \longrightarrow \Lambda$ by

$$
\theta\left(S_{1}\right)=S_{1}, \theta\left(S_{q}\right)=S_{q-1} .
$$

Let us consider the following calculation. First, observe that $\max \left\{d\left(x_{q}, \theta x_{p}\right), d\left(\theta x_{q}, x_{p}\right)\right\} \neq 0 \Leftrightarrow[(p>2 \wedge q=1) \vee(p$ $>q>1)]$.

For each $p \in \mathbb{N}, p>2$, we have

$$
\begin{gathered}
\frac{\mathbb{H}\left(\Theta S_{p}, \Theta S_{1}\right)}{d\left(S_{p}, \theta S_{p}\right) d\left(S_{p}, \theta S_{1}\right)+d\left(S_{1}, \theta S_{1}\right) d\left(S_{1}, \theta S_{p}\right) / \max \left\{d\left(S_{p}, \theta S_{1}\right) d\left(S_{1}, \theta S_{p}\right)\right\}} \\
=\frac{S_{p-1}^{2}}{S_{p}^{2} \cdot S_{1}^{2}+S_{1}^{2} \cdot S_{p-1}^{2} / S_{p}^{2}} \leq \frac{S_{p-1}^{2}}{S_{p}^{2}}=\frac{(p-1)^{2}}{(p+1)^{2}}<e^{-0.02} .
\end{gathered}
$$

For each $p, q \in N, p>q>1$, we have

$$
\begin{gathered}
\frac{\mathbb{H}\left(\Theta S_{p}, \Theta S_{q}\right)}{d\left(S_{p}, \theta S_{p}\right) d\left(S_{p}, \theta S_{q}\right)+d\left(S_{q}, \theta S_{q}\right) d\left(S_{q}, \theta S_{p}\right) / \max \left\{d\left(S_{p}, \theta S_{q}\right) d\left(S_{q}, \theta S_{p}\right)\right\}} \\
=\frac{S_{p}^{2} S_{p-1}^{2}}{S_{p}^{2} S_{p-1}^{2}+S_{q}^{2} S_{p}^{2}} \leq \frac{S_{p}^{2}}{S_{q}^{2}+S_{p}^{2}}=\frac{(p(p-1))^{2}}{(q(q+1))^{2}+(p(p+1))^{2}}<e^{-0.02} .
\end{gathered}
$$

Multiplying 1.01 on both sides and taking log to the base $e$ on both sides, we get inequality (7) and also find that $\tau=$ $0.004365 \cdots$. Therefore, $\theta$ and $\Theta$ have a fixed point.

\section{Application to Force Damping Oscillation}

Throughout in this section, $\max \{d(h, \theta k), d(\theta h, k)\} \neq 0$.

Assume that an object of mass $m$ moves to and fro on the $x$-axis around an equilibrium position $x=0$ (see Figure 1). The object has position $x(t)$ at time $t$. It undergoes a force due to a spring:

$$
F_{s}=-k x,
$$

Furthermore, a damping force that resists the movement of the object is shown:

$$
F_{d}=b \frac{d x}{d t}
$$

Now, by the second law of motion,

$$
F_{\text {net }}=m \frac{d^{2} x}{d t^{2}}
$$

where $m, k$, and $b$ are all positive constants. Up to that feature, the system is simply the damped harmonic oscillator.

Now, suppose the additional time-dependent force $f(t)$ is applied to the object. Then, by Newton's second law,

$$
\begin{aligned}
-k x-b \frac{d x}{d t}+f(t) & =m \frac{d^{2} x}{d t^{2}}, m \frac{d^{2} x}{d t^{2}}+b \frac{d x}{d t}+k x \\
& =f(t), x(0)=0, x^{\prime}(0)=0 .
\end{aligned}
$$

The problem (43) can be written in the form of the Fredholm integral equation: 


$$
v\left(t_{1}\right)=\int_{0}^{1} G((\beta, s)) \Theta_{1}\left(t_{1}, v\left(s_{1}\right)\right) d s_{1}, t_{1} \in[0,1] .
$$

Here, Green's function for critically damping oscillation is defined as

$$
\mathscr{G}\left(\beta, s_{1}\right)= \begin{cases}-s_{1} e^{\tau(\beta-t)}, & 0 \leq s_{1} \leq \beta \leq 1, \\ -t e^{\tau(t-\beta)}, & 0 \leq \beta \leq s_{1} \leq 1\end{cases}
$$

where $\tau$ can be found in terms of $m, b$, and $k$.

Let $\Lambda=C[0,1]$ be the set of all continuous functions defined on $[0,1]$. For $u \in C([0,1])$, define the supremum norm as

$$
\|v\|=\left(\sup _{t_{1} \in[0, t]}\left|v\left(t_{1}\right)\right|\right) e^{-\tau}
$$

Let $C([0,1], \mathbb{R})$ be endowed with the $b$-metric:

$$
d(v, \zeta)=\sup _{t_{1} \in[0,1]}\left\|v\left(t_{1}\right)-\zeta\left(t_{1}\right)\right\| e^{-\tau}
$$

With these contexts, $C([0,1], R,\|\|$.$) becomes the Banach$ space. We give the following theorem.

Theorem 18. Assume the assumptions given below hold.

$\left(A_{1}\right)$ There exists a continuous function $\Theta_{1}:[0,1] \times[0$, $1] \longrightarrow[0, \infty)$ and for $t_{1}, s_{1} \in[0, t]$ such that

$$
\begin{aligned}
& \left|\Theta_{1}\left(t_{1}, s_{1}, u\right)-\Theta_{1}\left(t_{1}, s_{1}, v\right)\right| \leq \sqrt{\frac{e^{-\tau}}{s}} M_{s}(h, k) \\
& M_{s}(h, k)=\sqrt{\frac{e^{-\tau}}{s}\left\{\frac{d(h, \theta h) d(h, \theta k)+d(k, \theta k) d(k, \theta h)}{\max \{d(h,), d(\theta h, k)\}}\right\}}
\end{aligned}
$$

where $\theta: C([0,1]) \longrightarrow C([0,1])$ is any function.

$\left(A_{2}\right) \int_{0}^{1} G\left(\beta, s_{1}\right) d s_{1} \leq 1$, for some $\tau>0$.

Then, the problem $(43)$ has a solution in $C([0,1])$.

Proof. Define $\Theta: C([0,1]) \longrightarrow C([0,1])$ by

$$
\Theta v\left(t_{1}\right)=\int_{0}^{1} G\left(\left(\beta, s_{1}\right)\right) \Theta_{1}\left(t_{1}, v\left(s_{1}\right)\right) d s_{1}, \quad t_{1} \in[0, t] .
$$

We have

$$
\begin{aligned}
|\Theta v-\Theta \zeta|= & \int_{0}^{1} G\left(\left(\beta, s_{1}\right)\right) \mid \Theta_{1}\left(t_{1}, s_{1}, v\left(s_{1}\right)\right) d s_{1} \\
& -\Theta_{1}\left(t_{1}, s_{1}, \zeta\left(s_{1}\right)\right)\left|d s_{1},\right| \Theta v-\Theta \zeta \mid \\
\leq & \frac{1}{\sqrt{s}} \int_{0}^{1} G\left(\beta, s_{1}\right) \sqrt{e^{-\tau} M_{s}(h, k)} d s_{1} \\
\leq & \frac{1}{\sqrt{s}} G\left(\beta, s_{1}\right) \sqrt{e^{-\tau} M_{s}(h, k)} d s_{1} \\
\leq & \frac{1}{e^{-2 \tau} \sqrt{s}} \sqrt{e^{-\tau} M_{s}(h, k)} \int_{0}^{1}\left(\beta, s_{1}\right) d s_{1} \\
\leq & \frac{1}{\sqrt{s}} \sqrt{e^{-\tau} M_{s}(h, k)} .
\end{aligned}
$$

Squaring both sides, we get

$$
\left|\Theta_{v}-\Theta \zeta\right|_{2} \leq \frac{1}{2} e^{-\tau}\left\{\frac{d(h, \theta h) d(h, \theta k)+d(k, \theta k) d(k, \theta h)}{\max \{d(h, \theta k), d(\theta h, k)\}}\right\}
$$

Multiplying on both sides $e^{-2 \tau}$ and taking sup, we get

$$
s d\left(\Theta_{v}, \Theta \zeta\right) \leq e^{-2 \tau}\left\{\frac{d(h, \theta h) d(h, \theta k)+d(k, \theta k) d(k, \theta h)}{\max \{d(h, \theta k), d(\theta h, k)\}}\right\}
$$

By taking $F(v)=\ln (v)$ from Theorem 12, the integral equation (44) has a common solution.

Similarly, we can apply our theorem for the existence of underdamping oscillation and overdamping oscillation.

\section{Application to Infinite Systems of Fractional Order Differential Equations}

Now, we have to derive sufficient conditions for the solutions in space $\mathrm{c}$ to the following nonlinear infinite systems of fractional order differential equations:

$$
\left\{\begin{array}{l}
\vartheta_{\alpha_{i}}=\frac{e^{-\tau}}{b(t)} b_{i}(t) \vartheta_{i}+\theta_{i}\left(\vartheta, \eta_{1}, \eta_{2}, \cdots\right), \alpha_{i} \in(0,1), \\
\vartheta^{\beta_{j}}=\frac{e^{-\tau}}{b(t)} b_{j}(t) \vartheta_{j}+\theta_{j}\left(\vartheta, \vartheta_{1}, \vartheta_{2}, \cdots\right), \beta_{j} \in(0,1),
\end{array}\right.
$$

with the initial condition $\vartheta_{0}=\vartheta_{i}^{0}$, where $t \in J, i, j=1,2 \cdots$, and $\tau$ is the positive real number. $J$ is any fixed interval on the real line. Let $\Lambda=\mathrm{c}$ be the space of all real sequences whose limit is finite.

$$
\|\eta\|=\left(\sup \left|\eta_{i}\right|\right) e^{-\tau}
$$


Let $(\mathbf{c}, \mathbb{R})$ be endowed with the $b$-metric:

$$
d(\eta, \vartheta)=\sup \left\|\eta_{i}-\vartheta_{i}\right\| e^{-\tau} .
$$

Theorem 19. Assume the assumptions given below hold.

$\left(A_{1}\right) \vartheta_{0}=\vartheta_{i}^{0} \in \mathbf{c} . \theta=\left(\theta_{1}, \theta_{2}, \cdots\right): J \times \mathbf{c} \longrightarrow \mathbf{c}$.

$\left(A_{2}\right) b_{i}(t)$ represents the continuous function on $J$.

$\left(A_{3}\right)$

$$
\begin{aligned}
& \left|\theta_{i}(t, \eta)-\theta_{i}(t, \vartheta)\right| \\
& \quad \leq \frac{e^{-\tau}}{2 s}\left\{\frac{\left(d\left(\eta, \theta_{j} \eta\right) d\left(\eta, \theta_{j} \vartheta\right)+d\left(\vartheta, \theta_{j} \vartheta\right) d\left(\vartheta, \theta_{j} \eta\right)\right)}{\eta\left\{d\left(\eta, \theta_{j} \vartheta\right), d\left(\theta_{j} \eta, \vartheta\right)\right\}}\right\}^{1 / 2},
\end{aligned}
$$

where $\eta=\left(\eta_{1}, \eta_{2}, \eta_{3} \cdots\right)$ and $\vartheta=\left(\vartheta_{1}, \vartheta_{2}, \vartheta_{3} \cdots\right)$.

$\left(A_{4}\right)$

$$
\begin{aligned}
& \left|b_{i}(t) \eta_{i}-b_{j}(t) \vartheta_{i}\right| \\
& \quad \leq \frac{b(t)}{2 s}\left\{\frac{\left(d\left(\eta, \theta_{j} \eta\right) d\left(\eta, \theta_{j} \vartheta\right)+d\left(\vartheta, \theta_{j} \vartheta\right) d\left(\vartheta, \theta_{j} \eta\right)\right)}{\eta\left\{d\left(\eta, \theta_{j} \vartheta\right), d\left(\theta_{j} \eta, \vartheta\right)\right\}}\right\}^{1 / 2},
\end{aligned}
$$

then, the infinite systems of fractional order differential (53) have a common solution in $\boldsymbol{c}$.

Proof. Define $h_{i}(t, \eta), h_{j}(t, \eta): J \times \mathbf{c} \longrightarrow \mathbf{c}$ by

$$
\left\{\begin{array}{l}
h_{i}(t, \eta)=\frac{e^{-\tau}}{b(t)} b_{i}(t) \eta_{i}+\theta_{i}\left(\eta, \eta_{1}, \eta_{2}, \cdots\right), \\
h_{j}(t, \vartheta)=\frac{e^{-\tau}}{b(t)} b_{j}(t) \eta_{j}+\theta_{j}\left(\vartheta, \vartheta_{1}, \vartheta_{2}, \cdots\right) .
\end{array}\right.
$$

We have

$$
\begin{aligned}
\left|h_{i}(t, \eta)-h_{i}(t, \vartheta)\right| \\
=\mid \frac{e^{-\tau}}{b(t)} b_{i}(t) \eta_{i}+\theta_{i}\left(\eta, \eta_{1}, \eta_{2}, \cdots\right) \\
\quad-\frac{e^{-\tau}}{b(t)} b_{j}(t) \vartheta_{1}-\theta_{i}\left(\vartheta, \vartheta_{1}, \vartheta_{2}, \cdots\right) \mid, \\
\leq\left|\frac{e^{-\tau}}{b(t)} b_{i}(t) \eta_{i}-\frac{e^{-\tau}}{b(t)} b_{j}(t) \vartheta_{j}\right| \\
\quad+\left|\theta_{i}\left(\eta, \eta_{1}, \eta_{2}, \cdots\right)-\theta_{j}\left(\vartheta, \vartheta_{1}, \vartheta_{2}, \cdots\right)\right|, \\
\leq \frac{e^{-\tau}}{2 s}\left\{\frac{d\left(\eta, \theta_{j} \eta\right) d\left(\eta, \theta_{j} \vartheta\right)+d\left(\vartheta, \theta_{j} \vartheta\right) d\left(\vartheta, \theta_{j} \eta\right)}{\max \left\{d\left(\eta, \theta_{j} \vartheta\right), d\left(\theta_{j} \eta, \vartheta\right)\right\}}\right\}^{1 / 2} \\
\quad+\frac{e^{-\tau}}{2 s}\left\{\frac{d\left(\eta, \theta_{j} \eta\right) d\left(\eta, \theta_{j} \vartheta\right)+d\left(\vartheta, \theta_{j} \vartheta\right) d\left(\vartheta, \theta_{j} \eta\right)}{\eta\left\{d\left(\eta, \theta_{j} \vartheta\right), d\left(\theta_{j} \eta, \vartheta\right)\right\}}\right\}^{1 / 2} .
\end{aligned}
$$

Squaring both sides, we get

$$
\begin{aligned}
& \left|h_{i}(t, \eta)-h_{i}(t, \vartheta)\right|^{2} e^{-\tau} \\
& \quad \leq \frac{e^{-2 \tau}}{s^{2}}\left\{\frac{d\left(\eta, \theta_{j} \eta\right) d\left(\eta, \theta_{j} \vartheta\right)+d\left(\vartheta, \theta_{j} \vartheta\right) d\left(\vartheta, \theta_{j} \eta\right)}{\max \left\{d\left(\eta, \theta_{j} \vartheta\right), d\left(\theta_{j} \eta, \vartheta\right)\right\}}\right\} .
\end{aligned}
$$

Taking the supremum, we get

$$
\begin{aligned}
s^{2} d & \left(h_{i}(t, \eta)-h_{i}(t, \vartheta)\right) \\
& \leq e^{-\tau}\left\{\frac{d\left(\eta, \theta_{j} \eta\right) d\left(\eta, \theta_{j} \vartheta\right)+d\left(\vartheta, \theta_{j} \vartheta\right) d\left(\vartheta, \theta_{j} \eta\right)}{\max \left\{d\left(\eta, \theta_{j} \vartheta\right), d\left(\theta_{j} \eta, \vartheta\right)\right\}}\right\} .
\end{aligned}
$$

By taking $F(v)=\ln (v)$ from Theorem 12 , the infinite systems of fractional order differential equation (53) have a common solution.

\section{Application to Functional Equations}

In this section, we study the solvability of functional equations using the established fixed point theorem.

Let $\Lambda_{1}$ and $\Lambda_{2}$ be Banach spaces, $\Theta_{1} \subset \Lambda_{1}, \Theta_{2} \subset \Lambda_{2}$, and $\mathbb{R}$ be the field of real numbers. Suppose $\Lambda=B\left(\Theta_{1}\right)$ represents the set of all functions defined on $\Theta_{1}$ which is bounded and real valued. Define $d: \Lambda \times \Lambda \longrightarrow \mathbb{R}^{+}$by

$$
d(v, \zeta)=|v-\zeta|^{2} .
$$

Then, $(\Lambda, d, s)$ is called the $b$-metric space. Consider the system of functional equations given as

$$
\begin{cases}p(v)=\sup _{\zeta \in \Theta_{2}}\left\{g(v, \zeta)+\Phi_{1}(v, \zeta, Q(\tau(v, \zeta)))\right\}, & v \in \Theta_{1}, \\ q(v)=\sup _{\zeta \in \Theta_{2}}\left\{g(v, \zeta)+\Phi_{2}(v, \zeta, Q(\tau(v, \zeta)))\right\}, & v \in \Theta_{1},\end{cases}
$$

Here, $g: \Theta_{1} \times \Theta_{2} \longrightarrow \mathbb{R}$ and $\Phi_{1}, \Phi_{2}: \Theta_{1} \times \Theta_{2} \times \mathbb{R} \longrightarrow$ $\mathbb{R}$ are functions which are bounded. $\Theta_{1}$ and $\Theta_{2}$ are the state and decision spaces, respectively. $\tau: \Theta_{1} \times \Theta_{2} \longrightarrow \Theta_{1}$ shows the transformation of the process, and $p(v)$ and $q(v)$ signify sup return functions with initial state $\Lambda$. Let $\Theta, \theta: B\left(\Theta_{1}\right)$ $\longrightarrow B\left(\Theta_{1}\right)$ be defined by

$$
\begin{cases}\Theta\left(h_{1}(v)\right)=\sup _{\zeta \in \Theta_{2}}\left\{g(v, \zeta)+\Phi_{1}(v, \zeta, P(\tau(v, \zeta)))\right\}, & v \in \Theta_{1}, \\ \theta\left(h_{1}(v)\right)=\sup _{\zeta \in \Theta_{2}}\left\{g(v, \zeta)+\Phi_{2}(v, \zeta, P(\tau(v, \zeta)))\right\}, & v \in \Theta_{1},\end{cases}
$$

Theorem 20. Let $\Phi_{1}, \Phi_{2}: \Theta_{1} \times \Theta_{2} \times \mathbb{R} \longrightarrow \mathbb{R}$ and $g: \Theta_{1} \times$ $\Theta_{2} \longrightarrow \mathbb{R}$ be continuous and bounded and satisfy the following assumption. 


$$
\begin{aligned}
& \left|\Phi_{1}(v, \zeta, h(v))-\Phi_{1}(v, \zeta, k(v))\right| \\
& \quad \leq \frac{e^{-\tau}}{\sqrt{s}}\left\{\sqrt{\frac{d(h, \theta h) d(h, \theta k)+d(k, \theta k) d(k, \theta h)}{\max \{d(h, \theta k), d(\theta h, k)\}}}\right\},
\end{aligned}
$$

for all $v \in \Theta_{1}$ and $\zeta \in \Theta_{2}$. Then, the system of functional equation (63) has a common solution.

Proof. Let $\delta$ be an arbitrary positive number, $v \in \Theta_{1}$, and $h_{1}, h_{2} \in B(\Lambda)$; then, there exist $\zeta_{1}, \zeta_{2} \in \Theta_{2}$ such that

$$
\begin{aligned}
& \Theta\left(h_{1}(v)\right)<g\left(v, \zeta_{1}\right)+\Phi_{1}\left(v, \zeta_{1}, h_{1}\left(\tau_{1}\right)\right)+\delta, \\
& \Theta\left(h_{2}(v)\right)<g\left(v, \zeta_{2}\right)+\Phi_{1}\left(v, \zeta_{2}, h_{2}\left(\tau_{2}\right)\right)+\delta, \\
& \Theta\left(h_{1}(v)\right) \geq g\left(v, \zeta_{2}\right)+\Phi_{1}\left(v, \zeta_{2}, h_{1}\left(\tau_{2}\right)\right), \\
& \Theta\left(h_{2}(v)\right) \geq g\left(v, \zeta_{1}\right)+\Phi_{1}\left(v, \zeta_{1}, h_{2}\left(\tau_{1}\right)\right) .
\end{aligned}
$$

Using equations (66) and (69),

$$
\begin{aligned}
\Theta & \left(h_{1}(v)\right)-\Theta\left(h_{2}(v)\right) \\
& \leq \Phi_{1}\left(v, \zeta_{1}, h_{1}\left(\tau\left(v, \zeta_{1}\right)\right)\right)-\Phi_{1}\left(v, \zeta_{1}, h_{2}\left(\tau\left(v, \zeta_{1}\right)\right)\right)+\delta \\
& \leq\left|\Phi_{1}\left(v, \zeta_{1}, h_{2}\left(\tau\left(v, \zeta_{1}\right)\right)\right)-\Phi_{1}\left(v, \zeta_{1}, h_{1}\left(\tau\left(v, \zeta_{1}\right)\right)\right)\right|+\delta \\
& \leq \frac{e^{-\tau}}{\sqrt{s}}\left\{\sqrt{\frac{d(h, \theta h) d(h, \theta k)+d(k, \theta k) d(k, \theta h)}{\max \{d(h, \theta k), d(\theta h, k)\}}}\right\}+\delta .
\end{aligned}
$$

It follows that

$$
\begin{aligned}
& \Theta\left(h_{1}(v)\right)-\Theta\left(h_{2}(v)\right) \\
& \quad \leq \frac{e^{-\tau}}{\sqrt{s}}\left\{\sqrt{\frac{d(h, \theta h) d(h, \theta k)+d(k, \theta k) d(k, \theta h)}{\max \{d(h, \theta k), d(\theta h, k)\}}}\right\}+\delta .
\end{aligned}
$$

Similarly, using equations (67) and (68), we have

$$
\begin{aligned}
& \Theta\left(h_{2}(v)\right)-\Theta\left(h_{1}(v)\right) \\
& \quad \leq \frac{e^{-\tau}}{\sqrt{s}}\left\{\sqrt{\frac{d(h, \theta h) d(h, \theta k)+d(k, \theta k) d(k, \theta h)}{\max \{d(h, \theta k), d(\theta h, k)\}}}\right\}+\delta .
\end{aligned}
$$

Using equations (71) and (72), we get

$$
\begin{aligned}
& \left|\Theta\left(h_{1}(v)\right)-\Theta\left(h_{2}(v)\right)\right| \\
& \quad \leq \frac{e^{-\tau}}{\sqrt{s}}\left\{\sqrt{\frac{d(h, \theta h) d(h, \theta k)+d(k, \theta k) d(k, \theta h)}{\max \{d(h, \theta k), d(\theta h, k)\}}}\right\}+\delta,
\end{aligned}
$$

for all $v \in \Theta_{1}$, and $\delta>0$ is arbitrary; therefore,

$$
\begin{aligned}
& s\left(\left|\Theta\left(h_{1}(v)\right)-\Theta\left(h_{2}(v)\right)\right|^{2}\right) \\
& \quad \leq e^{-2 \tau}\left(\left\{\sqrt{\frac{d(h, \theta h) d(h, \theta k)+d(k, \theta k) d(k, \theta h)}{\max \{d(h, \theta k), d(\theta h, k)\}}}\right\}\right)^{2} .
\end{aligned}
$$

Taking logarithms, we have

$$
\begin{aligned}
& \ln \left(s\left|\Theta\left(h_{1}(v)\right)-\Theta\left(h_{2}(v)\right)\right|^{2}\right) \\
& \quad \leq \ln \left(e^{-2 \tau}\left\{\sqrt{\frac{d(h, \theta h) d(h, \theta k)+d(k, \theta k) d(k, \theta h)}{\max \{d(h, \theta k), d(\theta h, k)\}}}\right\}\right)^{2} .
\end{aligned}
$$

After simple calculation, we get

$$
\begin{aligned}
2 \tau & +\ln \left(s d\left(\Theta\left(h_{1}(v)\right), \Theta\left(h_{2}(v)\right)\right)\right) \\
& \leq \ln \left(\frac{d(h, \theta h) d(h, \theta k)+d(k, \theta k) d(k, \theta h)}{\max \{d(h, \theta k), d(\theta h, k)\}}\right) .
\end{aligned}
$$

By taking $F(v)=\ln (v)$ from Theorem 12 , the system of functional equation (63) has a common solution.

\section{Data Availability}

No data were used to support the study.

\section{Conflicts of Interest}

We have no competing interests.

\section{Authors' Contributions}

All the authors contributed equally, and they read and approved the final manuscript for publication.

\section{Acknowledgments}

The third author would like to thank Prince Sultan University for funding this work through the research group Nonlinear Analysis Methods in Applied Mathematics (NAMAM) (Group Number RG-DES-2017-01-17).

\section{References}

[1] S. Czerwik, "Nonlinear set-valued contraction mappings in b -metric spaces," Atti Semin. Mat. Fis. Univ. Modena, vol. 46, pp. 263-276, 1998.

[2] I. A. Bakhtin, "The contraction mapping principle in quasimetric spaces in functional analysis," Ul yanovsk. Gos. Ped. Inst. Ul yanovsk, vol. 30, pp. 26-37, 1989.

[3] A. Azam, N. Mehmood, J. Ahmad, and J. S. Radenovic, "Multivalued fixed point theorems in cone b-metric spaces," Journal of inequalities and applications, vol. 58, no. 2, 2013.

[4] R. George, S. Radenovic, K. P. Reshma, and S. Shukla, "Rectangular b-metric space and contraction principles," Journal of 
Nonlinear Sciences and Applications, vol. 8, no. 6, pp. 10051013, 2015.

[5] V. Parvaneh, J. R. Roshan, and S. Radenovic, "Existence of tripled coincidence points in ordered b-metric spaces and an application to a system of integral equations," Fixed Point Theory and Applications, vol. 2013, no. 1, 2013.

[6] B. Popovic, S. Radenovic, and S. Shukla, "Fixed point results to tvs-cone b- metric spaces," Gulf Journal of Mathematics, vol. 1, pp. 51-64, 2013.

[7] S. Phiangsungnoen and P. Kumam, "Generalized Ulam-Hyers stability and well-posedness for fixed point equation via $\alpha$ admissibility," Journal of Inequalities and Applications, vol. 2014, no. 418, 2014.

[8] T. Abdeljawad, N. Mlaiki, H. Aydi, and N. Souayah, "Double controlled metric type spaces and some fixed point results," Mathematics, vol. 6, no. 12, p. 320, 2018.

[9] H. Afshari, "Solution of fractional differential equations in quasi-b-metric and b-metric-like spaces," Advances in Difference Equations, vol. 2019, no. 285, 2019.

[10] H. Afshari, S. Rezapour, and N. Shahzad, "Absolute retractivity of the common fixed points set of two multifunctions," Topological Methods in Nonlinear Analysis, vol. 40, pp. 429436, 2012.

[11] B. Alqahtani, A. Fulga, and E. Karapınar, "Common fixed point results on an extended b-metric space," Journal of Inequalities and Applications, vol. 2018, Article ID 158, 2018.

[12] A. Mukheimera, N. Mlaikia, K. Abodayeha, and W. Shatanawi, "New theorems on extended b-metric spaces under new contractions," Nonlinear Analysis: Modelling and Control, vol. 24, no. $6,2019$.

[13] M. A. Geraghty, "On contractive mappings," Proceedings of the American Mathematical Society, vol. 40, no. 2, pp. 604-608, 1973.

[14] H. Afshari, H. H. Alsulami, and E. Karapinar, "On the extended multivalued Geraghty type contractions," Journal of Nonlinear Sciences \& Applications (JNSA), vol. 9, no. 6, pp. 4695-4706, 2016.

[15] D. Dukic, Z. Kadelburg, and S. Radenovic, "Fixed points of Geraghty-type mappings in various generalized metric spaces," Abstract and Applied Analysis, vol. 2011, 13 pages, 2011.

[16] Z. Liu, B. Xu, and S. M. Kang, "Two fixed point theorems of mappings satisfying contractive inequalities of integral type," International Journal of Pure and Applied Mathematics, vol. 90, pp. 85-100, 2014.

[17] M. Shoaib, M. Sarwar, and P. Kumam, "Multi-valued fixed point theorem via $\mathrm{F}$-contraction of Nadler type and application to functional and integral equations," Boletim da Sociedade Paranaense de Matemática, vol. 39, no. 4, pp. 83-95, 2020.

[18] M. Shoaib, T. Abdeljawad, M. Sarwar, and F. Jarad, "Fixed point theorems for multi-valued contractions in b-metric spaces with applications to fractional differential and integral equations," IEEE Access, vol. 7, pp. 127373-127383, 2019.

[19] M. S. Khan, "A fixed point theorem for metric spaces," Rendiconti dell'Istituto di Matematica dell'Università di Trieste, vol. 8, pp. 69-72, 1976.

[20] B. Fisher, "On a theorem of Khan," Rivista di Matematica della Università di Parma, vol. 4, pp. 135-137, 1978.

[21] H. Piri, S. Rahrovi, and P. Kumam, "Generalization of Khan fixed point theorem," Journal of Mathematics and Computer Science, vol. 17, no. 1, pp. 76-83, 2017.
[22] H. Piri, S. Rahrovi, H. Marasi, and P. Kumam, “A fixed point theorem for F-Khan-contractions on complete metric spaces and application to integral equations," Journal of Nonlinear Sciences and Applications, vol. 10, no. 9, pp. 4564-4573, 2017.

[23] A. Ullah, I. S. Khan, and N. Mehmood, "Fixed point and common fixed point results of $\mathrm{D}_{\mathrm{F}}-$ contractions via measure of non-compactness with applications," Communication in mathematics and applications, vol. 9, pp. 53-62, 2018.

[24] S. B. Nadler, "Multi-valued contraction mappings," Pacific Journal of Mathematics, vol. 20, no. 2, pp. 475-488, 1969.

[25] M. Cosentino and P. Vetro, "Fixed point results for F-contractive mappings of Hardy-Rogers-type," Univerzitet $u$ Nišu, vol. 28, pp. 715-722, 2014.

[26] D. Wardowski, "Fixed points of a new type of contractive mappings in complete metric spaces," Fixed Point Theory and Applications, vol. 94, 2012.

[27] M. Sgroi and C. Vetro, "Multi-valued F-contractions and the solution of certain functional and integral equations," Univerzitet $u$ Nišu, vol. 27, no. 7, pp. 1259-1268, 2013.

[28] M. Usman Ali, T. Kamran, and M. Postolache, "Solution of Volterra integral inclusion in b-metric spaces via new fixed point theorem," Nonlinear Analysis: Modelling and Control, vol. 22, 2017.

[29] R. Batra and S. Vashistha, "Fixed points of an F-contraction on metric spaces with a graph," International Journal of Computer Mathematics, vol. 91, no. 12, pp. 2483-2490, 2014.

[30] G. Minak, A. Helvac1, and I. Altun, "Ciric type generalized'F -contractions on complete metric spaces and fixed point results," Univerzitet u Nišu, vol. 28, no. 6, pp. 1143-1151, 2014.

[31] N.-A. Secelean, "Iterated function systems consisting of $\mathrm{F}$ -contractions," Fixed Point Theory and Applications, vol. 2013, no. 1, 2013.

[32] D. Paesano and C. Vetro, "Multi-valued F-contractions in 0complete partial metric spaces with application to Volterra type integral equation," Revista de la Real Academia de Ciencias Exactas, Fisicas y Naturales. Serie A. Matematicas, vol. 108, no. 2, pp. 1005-1020, 2014.

[33] M. Cosentino, M. Jleli, B. Samet, and C. Vetro, "Solvability of integrodifferential problems via fixed point theory in b-metric spaces," Fixed Point Theory and Applications, vol. 2015, Article ID 70, 2015.

[34] Z. Liu, X. Li, S. M. Kang, and S. Y. Cho, "Fixed point theorems for mappings satisfying contractive conditions of integral type and applications," Fixed Point Theory and Applications, vol. 2011, no. 1, 2011. 